\title{
ANTECEDENTES, DESCRIPCIÓN, POTENCIA DEL TREN INFERIOR Y PLIOMETRÍA EN FÚTBOL SALA
}

\author{
BACKGROUND, DESCRIPTION, LOWER TRAIN POWER AND PLYOMETRICS IN \\ FUTSAL
}

Cómo citar: Cepeda, C., Gamboa, F., \& Sanabria, Y. (2019). Antecedentes, descripción, potencia del tren inferior y pliometría en fútbol sala. R. Actividad fis. y deporte. 6 (1): 165-178.

Artículo de acceso abierto publicado por: Revista Digital: Actividad Física y Deporte, bajo una licencia Creative Commons CC BY-NC 4.0.

\section{Cristian Leonardo Cepeda Barajas}

Licenciado en Educación Física, Recreación y Deporte

- UPTC. Maestrante en Pedagogía de la Cultura

Física.

cristiancepeda14@gmail.com

\section{Franky Smith Gamboa Agudelo}

Licenciado en Educación Física, Recreación y Deporte - UPTC. Maestrante en Pedagogía de la Cultura Física.

Franky.gamboaa@gmail.com

\section{Yofre Danilo Sanabria Arguello}

Dr. (Ph.D) (c) Educación Deportiva y Ciencias del Deporte. Mg. Pedagogías de la Cultura Física. Docente Universidad Pedagógica y Tecnológica de Colombia-UPTC.

yofre.sanabria@uptc.edu.co

\section{RESUMEN}

Introducción: En el fútbol sala la potencia se ha constituido en uno de los factores determinantes, para lograr una dinámica colectiva que se percibe en las diferentes competiciones. Objetivo general: Aplicar una revisión bibliográfica rigurosa con la finalidad de indagar e identificar la influencia del método de entrenamiento pliométrico sobre la potencia del tren inferior en deportistas de fútbol sala. Metodología: La investigación tiene como fin destacar los efectos más relevantes en las investigaciones revisadas para dar una herramienta más eficaz a los profesionales de este deporte, en el momento de seleccionar un método de entrenamiento como medio para desarrollar la potencia del tren inferior. Resultados: Estos fueron determinados por los trabajos de campo realizados por las diversas investigaciones sobre este tema, que orientó la información necesaria para facilitar el desarrollo de la potencia. Conclusiones: Se extraen de los trabajos de campo aplicados y acompañados de sus análisis estadísticos, que facilitaron, obtener la información adecuada y pertinente, en cuanto a los efectos que produce el método de entrenamiento pliométrico sobre la potencia de tren inferior de las unidades de estudio. 
Palabras clave: Pliometría, fútbol sala, potencia de tren inferior.

\section{ABSTRACT}

Introduction: In futsal, the power has become one of the determining factors, to achieve a collective dynamic that is perceived in the different competitions. Course objective: Apply a rigorous bibliographic review with the purpose of investigating and identifying the influence of the plyometric training method on the power of the lower train in indoor soccer athletes. Methodology: The research aims to highlight the most relevant effects in the reviewed research to give a more effective tool to the professionals of this sport, when selecting a training method as a means to develop the power of the lower train. Results: These were determined by the field work carried out by the various investigations on this subject, which provided the necessary information to facilitate the development of power. Conclusions: They are extracted from the field work applied and accompanied by their statistical analyzes, which facilitated obtaining adequate and pertinent information, regarding the effects produced by the plyometric training method on the lower train power of the study units.

Key words: Plyometrics, futsal, lower train power.

\section{INTRODUCCIÓN}

El deporte, en cuanto a tecnología y métodos de entrenamiento, ha tenido gran evolución, buscando ser cada vez más específico en el desarrollo de las capacidades de los deportistas desde cualquier etapa de su formación deportiva. Por tanto, en las diferentes modalidades deportivas individuales y colectivas es necesario trabajar las capacidades condicionales (Fuerza, velocidad, resistencia, flexibilidad y las coordinativas), por esta razón, uno de las causas, a nivel físico, que más se ha estudiado actualmente y que ha demostrado gran relevancia y utilidad en la planificación del entrenamiento de las diversas disciplinas deportivas es la fuerza. De ahí, que en el fútbol sala, la potencia se ha constituido como uno de los factores determinantes para lograr una dinámica colectiva que se percibe en las diferentes competiciones. Siendo que la "fuerza ocupa un lugar esencial para cualquier ser humano, ya sea como capacidad física fundamental, limitante del rendimiento, o bien para garantizar la realización de cualquier acción motora" (Siff \& Verkhoshansky, 2000).

Por tal motivo, se puede evidenciar que, el Fútbol sala, requiere de potencia en el tren inferior para mantener un rendimiento deportivo óptimo en las competencias donde participen, al hablar de "potencia se refiere al producto entre la fuerza aplicada y la velocidad de la acción realizada" (Herzong \& Ait- Haddou, 2003). Esto permite tener un 
mejor desempeño durante el juego y lograr mejorar el rendimiento de los deportistas en las diferentes situaciones de juego.

El fútbol sala es un deporte que se caracteriza por el desarrollo de situaciones de juego de corta duración y de intensidad máxima notables para el marcador que activa primordialmente cualidades de fuerza $y$ velocidad, según, Bomba, (2003). En efecto los desplazamientos con más explosividad y mayor destreza física son clave importante en el desarrollo del juego, debido a que la movilidad individual más eficaz y eficiente logra concretar un mejor trabajo en equipo. En consecuencia, las acciones cortas y explosivas resultan ser las más determinantes en un partido de fútbol sala. Existen dos derivaciones de la potencia, que se hacen necesarias para que un deportista de fútbol sala logre mantener un rendimiento adecuado y pertinente y que corresponden a las siguientes:

1- Potencia de Arranque: Según (González, J., 2000) un jugador debe ser rápido percibiendo un estímulo y procesando la información, al mismo tiempo debe generar la máxima fuerza en el menor tiempo, para proporcionar una respuesta inmediata y efectiva.

2- Potencia de aceleración y desaceleración: Según (González, J., 2000) es conseguir la más alta frecuencia de zancada, la menor fase de contacto posible cuando la pierna toma contacto con el piso, y la más alta propulsión cuando la pierna empuja en contra del piso, para lograr un potente impulso hacia delante. Además, debe ser capaz de frenar el movimiento con la menor inercia posible, determinante para ejecutar un cambio de ritmo con eficacia. En este sentido, el presente documento está encaminado a determinar, a través del rastreo bibliográfico de investigaciones desarrolladas, las adaptaciones inducidas del método de entrenamiento pliométrico sobre la potencia del tren inferior en deportistas practicantes del fútbol sala. Esto con el objetivo de concluir, sí, existe un efecto positivo en la potencia del tren inferior de los sujetos objeto de estudio. Y así, poder contribuir con los procesos formativos de los profesionales que dirigen en el deporte, con el fin de generar nuevas herramientas útiles y de bajo costo, para mejorar la condición de sus deportistas, y así mismo, aumentar la posibilidad de consecución de altos logros en estos deportistas.

\section{METODOLOGÍA}

El presente artículo es una revisión bibliográfica, basada en el análisis de los resultados de investigaciones ya terminadas, que corresponden a la aplicación del método de entrenamiento pliométrico, como medio para trabajar la potencia del tren inferior en practicantes del fútbol sala. Este documento es de carácter descriptivo, porque busca establecer por medio de la revisión bibliográfica, los efectos que genera la utilización del método de entrenamiento pliométrico en la potencia del tren inferior de los practicantes del deporte ya mencionado. 
La presente revisión se llevó a cabo según interés de los autores, para poder comparar y analizar los resultados de investigaciones relacionadas con pliometría, potencia de tren inferior y fútbol sala.

\section{CONCEPTUALIZACIÓN}

Entrenamiento deportivo: Mora, V. (1995) Plantea que: es un proceso planificado y complejo que organiza cargas de trabajo progresivamente crecientes destinada a estimular los procesos fisiológicos de súper compensación del organismo. De tal manera, que el entrenamiento deportivo busca fortalecer el trabajo de las capacidades específicas del deporte, con el fin de mejorar la forma deportiva de los participantes.

Capacidades físicas: Villar, A. (1985) define las capacidades físicas como: los factores que determinan la condición física de un individuo y lo orientan para la realización de una determinada actividad física, posibilitando mediante el entrenamiento que un sujeto desarrolle al máximo su potencial físico. Gutiérrez, F. (2010) dice que se dividen en cuatro: 1.- Resistencia, 2.- Velocidad, 3.Flexibilidad y 4.- Fuerza, que son complementadas y desarrolladas para mejorar el rendimiento físico.

Las capacidades físicas son las que determinan el estado físico de un individuo y pueden desarrollarse mediante el entrenamiento. Estas capacidades físicas nos dan la posibilidad de ser efectivos en las tareas deportivas que realizamos, por tanto, deben someterse a un entrenamiento constante para poder ver mejoras de las capacidades en mención.

Pliometría: A continuación, se sustenta uno de los conceptos importantes para el desarrollo de salto en los jugadores de fútbol sala (Zatsiorski, 1989) fue quien utilizó en 1966 por primera vez, el vocablo pliométrico. El autor buscaba con este término expresar el alto grado de tensión que producía un grupo muscular en la sucesiva y veloz secuencia de tensión excéntrica-contracción concéntrica. Paralelamente, a esto, el profesor Rodolfo Margaría realizaba en Milán investigaciones fisiológicas y biomecánicas de este nuevo tipo de movimiento, considerando al conjunto del grupo muscular como un todo, según, Chu, D.(1993): El verdadero término pliométrico fue acuñado por primera vez: Wilt, F. (1975) un entrenador de atletismo de los Estados Unidos, quien indica que es un término de raíz latina, plyo+metrics se interpreta que quiere decir: Aumentos medibles. Independientemente, a ésto algunos autores Soviéticos venían tocando este término desde la década del 60 con los resultados del mundialmente conocido Valery Brumal.

De igual forma, la pliometría es un método de entrenamiento de la fuerza reactiva utilizada para mejorar el rendimiento deportivo, incrementando la velocidad o la Saltabilidad de los deportistas. Es un método específico de preparación de la fuerza dirigida al desarrollo de la fuerza explosiva muscular y de la capacidad reactiva del sistema neuromuscular (Delgado, P., 2011). 
Mas autores como: (García Manso) define la pliometría como: "aquellos ejercicios en que el músculo es cargado con una contracción excéntrica (estiramiento), seguido inmediatamente por una contracción concéntrica (acortamiento). En términos fisiológicos, ha sido demostrado que un músculo que es estirado antes de una contracción, se contraerá más fuerte y rápido".

Potencia: La potencia se puede definir como la máxima cantidad de trabajo o de tensión muscular que se puede desarrollar por una unidad de tiempo, o el producto de la fuerza por la velocidad" (Croin \& Sleivert, 2005). Como menciona el autor el trabajo de los grupos musculares durante el proceso deportivo es importante, además el desarrollo adecuado de la potencia en el tren inferior en jugadores de fútbol sala optimiza la calidad de los saltos del deportista, y sube los niveles de efectividad durante las competencias.

Hace parte de la fuerza (Hernández \& García, 2012). Indica que esta capacidad física permite optimizar el rendimiento en las acciones explosivas y rápidas de corta duración, que necesitan una gran potencia muscular que permite aplicar gran cantidad de fuerza en acciones deportivas.

Fuerza explosiva: La fuerza explosiva puede definirse como el resultado de la relación entre la fuerza producida y el tiempo utilizado para ello: González, J. (2000). Capacidad que permite generar una mayor explosividad durante el juego. Por otro lado:
Cerafin, (1993). Es aquella que intenta vencer una resistencia no límite, pero a una velocidad máxima, es más habitual en deportes acíclicos tales como: Saltos, remates de voleibol, lanzamientos, entre otros. Los gestos explosivos son típicos de movimientos acíclicos, en que la culminación del ciclo de movimiento no da comienzo a otro ciclo de movimiento.

Fútbol sala: Es un deporte colectivo con gran similitud al fútbol, con diferencias respecto al terreno de juego, medidas de la cancha, medidas de la portería, peso y tamaño del balón y algunas reglas de juego. Se juega entre dos equipos con 5 jugadores cada uno.

El objetivo del juego es: Introducir el balón en la portería del contrario (esto se llama anotar un Gol o un tanto), y evitar que el contrario obtenga el balón y lo introduzca en tu portería. El equipo que haya marcado el mayor número de goles durante un partido será el ganador. Sí, ambos equipos marcan el mismo número de goles o no marcan ninguno, el partido terminará en empate. Dentro de la cancha el portero es el único jugador que puede tocar el balón con sus manos, en su área de meta.

El resto de los jugadores juegan con sus pies e incluso pueden tocar el balón con cualquier parte del cuerpo excepto los brazos y manos, el terreno de juego debe ser de: Asfalto, madera o cemento pulimentado, sin declive, ni resbaladizo. El terreno debe ser rectangular, con un largo entre 28 y $40 \mathrm{~m}$ (36 y $40 \mathrm{~m}$ para partidos internacionales), y su ancho entre 16 y 20 m (18 y 20 m para 
partidos internacionales). La superficie de juego deberá ser lisa.

\section{Resultado de la revisión bibliográfica frente a la pliometría como método de entrenamiento para mejorar la potencia en jugadores de fútbol sala.}

Actualmente, existen muchos trabajos, estudios y libros en todo el mundo dedicados al método de entrenamiento pliométrico, por ello se refleja la importancia que éste tiene para la preparación de las distintas modalidades deportivas. El considerado padre de la pliometría Yuri Verkhoshansky, asociada la pliometría a la potencia mecánica del tren inferior como una relevancia muy importante a nivel Internacional, llevándose a cabo una cadena de investigaciones documentadas en: Tesis, artículos y demás archivos relacionados con los programas de entrenamiento pliométricos, Ahora bien, considerando que en la mayoría de gestos deportivos toda contracción concéntrica va precedida de un estiramiento del músculo, daremos cuenta, de la importancia del trabajo de este ciclo estiramiento acortamiento.

Esta es la razón, por la que hoy en día está ampliamente aceptada la eficacia del método pliométrico como el artículo: “Metodología de entrenamiento pliométrico" (García, 2003). El objetivo de este estudio es profundizar en todos los aspectos referentes al entrenamiento pliométrico, incluyendo un breve repaso histórico, a fin de establecer unas directrices metodológicas que orienten a los entrenadores que pretendan incluir la pliometría dentro de su programación.

Por consiguiente, la literatura permite evidenciar una variedad de protocolos de entrenamiento pliométrico, por lo que es difícil concluir: ¿Cuál es el método más acorde? En la tabla a continuación se resumen las particularidades de los programas de entrenamiento utilizados en algunos estudios. 
Tabla 1. Característica de los programas de entrenamiento utilizados en algunos estudios, donde $S J=s q u a t$ jump, CMJ=Counter movement jum, RJ15= Repeat jump (15 segundos).

\begin{tabular}{|c|c|c|c|c|}
\hline AUTOR & $\begin{array}{l}\text { DURACIÓN DEL } \\
\text { PROGRAMA }\end{array}$ & $\begin{array}{l}\text { ALTURA DE CAIDA } \\
\text { EN LOS DJ }\end{array}$ & $\begin{array}{c}\text { NÚMERO DE } \\
\text { SALTOS/SESIÓN }\end{array}$ & $\begin{array}{c}\text { TEST EN LOS QUE SE } \\
\text { OBTUVO MEJORA }\end{array}$ \\
\hline Häkkinen, K., 1985. & $\begin{array}{l}24 \text { semanas (72 } \\
\text { sesiones). }\end{array}$ & No específica. & 100-200 (apoyos) & SJ $(P<0,01)$ \\
\hline $\begin{array}{l}\text { Brow, E., \& Mayhew, } \\
1986 .\end{array}$ & $\begin{array}{c}12 \text { semanas ( } 36 \\
\text { sesiones). }\end{array}$ & No específica. & 30. & $\operatorname{CMJ}(\mathrm{P}<0,05)$ \\
\hline Gemar, 1988. & $\begin{array}{l}8 \text { semanas ( } 16 \\
\text { sesiones). }\end{array}$ & No específica. & No específica & $\operatorname{CMJ}(P<0,05)$ \\
\hline $\begin{array}{l}\text { Wilson, Newton, } \\
\text { Murphy, \& Humphries, } \\
1993 .\end{array}$ & $\begin{array}{c}10 \text { semanas (30 } \\
\text { sesiones). }\end{array}$ & $20-80 \mathrm{~cm}$ & $30-60$ & $\operatorname{CMJ}(P<0,05)(10,33 \%)$ \\
\hline $\begin{array}{l}\text { Flarity, Shilstone, } \\
\text { Iglesia, \& Fisher, } 1997 .\end{array}$ & $\begin{array}{l}9 \text { semanas ( } 27 \\
\text { sesiones). }\end{array}$ & No específica. & No específica & Seargent $(P<0,05)$ \\
\hline $\begin{array}{l}\text { Diallo, Dore, Duche, \& } \\
\text { Van Praagh, } 2001 .\end{array}$ & $\begin{array}{c}10 \text { semanas ( } 30 \\
\text { sesiones). }\end{array}$ & $30-40 \mathrm{~cm}$. & 200-300 (apoyos). & $\begin{array}{c}\text { CMJ }(P<0,01)(11,6 \%) \\
\text { SJ }(P<0,01)(7,3 \%) \\
\text { RJ15 }(P<0,01)\end{array}$ \\
\hline $\begin{array}{l}\text { Matavulj, Kukolj, } \\
\text { Ugarkovic, Tihanyi, \& } \\
\text { Jaric, } 2001 .\end{array}$ & $\begin{array}{l}6 \text { semanas (18 } \\
\text { sesiones). }\end{array}$ & $50 \mathrm{~cm} 100 \mathrm{~cm}$. & 30 & $\begin{array}{l}\text { SJ }(P<0,05)(12,8 \%) \\
\text { SJ }(P<0,05)(13,3 \%)\end{array}$ \\
\hline $\begin{array}{l}\text { Spurrs, Murphy, \& } \\
\text { Watsford, } 2003 .\end{array}$ & $\begin{array}{l}6 \text { semanas ( } 15 \\
\text { sesiones). }\end{array}$ & No específica. & 127 (media) (apoyos). & $\operatorname{CMJ}(P<0,05)$ \\
\hline
\end{tabular}

Fuente: Metodología del Entrenamiento Pliométrico (García, D., et al., 2003, p. 6).

Los estudios expuestos en la tabla evaluaron programas de entrenamiento pliométrico por sí solo, pero de la misma manera fueron varias las investigaciones en las que se combinó la pliometría con otros métodos de entrenamiento. Siendo la pliometría un método sobresaliente en estas investigaciones, que manifiestan los efectos que genera este método sobre la potencia del tren inferior, así como también, se muestra que la eficacia de este método de entrenamiento depende de la duración del programa aplicado.

Así mismo, existe otro artículo que ayuda a soportar bases teóricas de la revisión realizada, como el realizado en Estados
Unidos, artículo publicado en la revista de Entrenamiento Deportivo mencionado: "Efectos de un programa de Entrenamiento pliométrico de seis semanas sobre la agilidad" (Miller, M., 2016). Los autores exponen en su investigación un programa de entrenamiento pliométrico de seis semanas para mejorar la agilidad de un atleta, donde se enfoca su estudio en un grupo que ejecuta el programa y otro grupo control. Donde se aplicaron: 1.Test de Ilinois, 2.- Test T y 3.-Un Test de placa para analizar los tiempos de reacción.

De igual manera, los resultados obtenidos en esta investigación revelan un efecto significativo de grupo ( $F 2, \quad 26=25,42$, $\mathrm{p}=0,0000$ ) para la medición de agilidad del 
Test T. También, fue encontrado un efecto significativo de grupo para la prueba de agilidad de Illinois $(F 2,26=27,24, p=0.000)$. Sin embargo, el grupo de entrenamiento pliométrico obtuvo tiempos post-test menores en comparación con el grupo de control en los tests de agilidad. De igual modo, fue encontrado un efecto significativo de grupo $(F 2,26=7,81, p=0,002)$ para el test en placa de fuerza. El grupo de entrenamiento pliométrico redujo el tiempo de contacto en el suelo en el post-test en comparación con el grupo control. Los resultados de este estudio demuestran que el entrenamiento pliométrico puede ser una técnica de entrenamiento eficaz para mejorar la agilidad de un atleta.

También, en Madrid, España encontramos un artículo en la revista europea de movimiento humano, la investigación, titulada: "Análisis de las adaptaciones inducidas por cuatro semanas de entrenamiento pliométrico" (García, D., 2005). El objetivo de nuestro trabajo fue valorar las adaptaciones inducidas por un programa de entrenamiento pliométrico del tren inferior de 4 semanas de duración (12 sesiones), aplicado a 9 estudiantes de educación física $(19.33 \pm 1.38$ años, $74.89 \pm 6.89 \mathrm{Kg})$, contando también, con un grupo control $(n=8)$. El grupo experimental obtuvo mejoras en la altura de diferentes saltos verticales (SJ, CMJ y Abalakov), fuerza máxima isométrica de extensión de rodilla y potencia pico en cicloergómetro (Test de Wingate). Si bien, ninguno de estos incrementos fue estadísticamente significativo. El grupo control no mostró mejoras en ningún test. El hecho que los incrementos no alcanzasen significación estadística pudo deberse a la escasa duración del programa aplicado, en comparación con los programas citados en la literatura.

Por otra parte, a nivel nacional se han encontrado investigaciones como “comparación entre las respuestas de potencia muscular producidas por entrenamiento pliométrico y banda elástica en jugadores juveniles de 16 años del Club Independiente Santa Fe" (Ramírez Mora, 2015). La investigación mencionada, busca comparar los efectos producidos por un Entrenamiento con Bandas Elásticas y la aplicación del método de Entrenamiento pliométrico para el desarrollo de la potencia en el fútbol, implementado con un grupo de deportistas juveniles del club Independiente Santa Fe, a partir de una investigación cuasi experimental, que maneja un grupo al que se le aplicará el método de entrenamiento Pliométrico y un grupo que trabajará con bandas elásticas.

Para ello, se elaboraron dos métodos de trabajo con 20 sesiones cada uno, realizando tres sesiones por semana, destacando el trabajo de Potencia; uno para ser aplicado con el entrenamiento Bandas Elásticas, y el otro, para ser desarrollado con el Entrenamiento Pliométrico, donde se evaluaron los deportistas con pre test y post test de Bosco, que mide la fuerza explosiva del tren inferior; con ésto se pretendió determinar el tiempo de aplicación y desarrollo de la fuerza la eficacia y eficiencia de los dos métodos de entrenamiento. Dentro de este orden de ideas el 
entrenamiento Con Bandas Elásticas y Pliométrico es eficiente para el desarrollo de la potencia en edades juveniles, no obstante, se debe tener en cuenta que el aumentar el número de semanas de entrenamiento permite que los resultados tengan una diferencia significativa más alta.

De igual manera, se evidenció de tal forma, porque mediante las comparaciones con los resultados de potencia máxima, que en el salto CMJ (contra movimiento) los resultados fueron superiores a los estudios analizados, dejando ver que la metodología de entrenamiento fue acertada tanto para el entrenamiento con Bandas Elásticas como para método Pliométrico. Así mismo, concluye con que este entrenamiento de potencia se debe realizar por lo menos 3 veces a la semana, con un día descanso entre sesión como se realizó en esta investigación, y no realizarse un día antes de la competencia, ni un día después de la misma, para no generar sobrecarga muscular que generaría lesiones.

En el mismo sentido, en la ciudad de Medellín se realizó un estudio titulado: la "fuerza explosiva en el futbolista profesional del club Deportivo Independiente Medellín durante la segunda temporada competitiva del 2006." (Ruíz De la Cruz \& Leal Ríos, 2007). Investigación que hace una caracterización de la fuerza analizando los principales medios y métodos modernos y busca realizar una comparación con la metodología trabajada por los futbolistas del club, para desarrollar las manifestaciones de fuerza explosiva, es así que, uno de los métodos aplicados para esta investigación es la pliometría, donde indica que este método es más eficaz para desarrollar la fuerza explosiva; de manera que, en una misma sesión se puede hacer un sólo tipo de flexión o combinar dos o tres ángulos de trabajo.

Por su parte, los autores de la investigación, hacen una clasificación donde indica que se puede trabajar pliometría baja, media y de alta intensidad, variando los ejercicios en cada una. En conclusión, la teoría consultada reitera la importancia de la caracterización del deporte, como eje central de la posterior planificación de las cargas de entrenamiento. El fútbol y las otras disciplinas deportivas tienen que aprovechar, todos los beneficios que arroja el entrenamiento de la fuerza. Los trabajos de musculación (cargas estandarizadas), la pliometría (baja, media y alta) y los trabajos específicos (tareas en espacios reducidos) favorecen o potencializan la mejora de la fuerza explosiva; $y$ en los jugadores del Deportivo Independiente Medellín se vió el reflejo del trabajo realizado, más que todo por el método de contrastes; en donde se mezclan estos diferentes regímenes de acción, en las sesiones de entrenamiento, permitiendo ganar al equipo una dinámica más colectiva.

De igual manera, se presentó una Revisión sistemática en Bogotá-Colombia, Revista digital: Actividad Física y Deporte; titulada: "Análisis de la evaluación de potencia en tren inferior" por los autores (Valero \& Suárez, 2018). Donde los autores buscan en varias bases de datos toda la información pertinente al tema, y teniendo como objetivo, identificar: Cuáles son los test más usados para la evaluación de la potencia, y 
específicamente, en tren inferior. El artículo para nuestra investigación es de suma importancia dada la recopilación de antecedentes y datos significativos; porque se aplican los diferentes test, dando resultados en tablas de datos soportadas, que nos brindan información base para nuestros postulados.

Por otro lado, se encontró un artículo a nivel regional titulado: "Métodos con pesas y pliometría: comparación de efectos para desarrollar potencia en jugadoras de futsal FIFA" (Mancipe, 2010), desarrollado en Tunja, Boyacá; en la que el autor, compara dos métodos de entrenamiento; uno tradicional y el otro el método Pliométrico, para así, ver la eficacia de cada uno aplicado sobre la potencia del tren inferior en dos grupos de fútbol sala. Como resultado se concluye que la investigación sirve para demostrar la eficacia de cada método, y que la combinación de los dos métodos genera buenos resultados, teniendo en cuenta, los aportes de cada método.

De igual manera, en lo correspondiente al nivel local se encuentra el estudio realizado por: Torrijos, Acosta, \& Benítez (2018): "Correlación entre la fuerza explosiva del tren inferior y la agilidad en el fútbol sala," que tuvo como objetivo correlacionar la fuerza explosiva del tren inferior y la agilidad en las jugadoras de fútbol sala de la Universidad Pedagógica y Tecnológica de Colombia sede seccional Chiquinquirá. Los resultados mostraron datos en lo que refiere a la agilidad de $(19,1 \pm 1,22 \mathrm{seg})$ y en la potencia se observó que en el test de Bosco en el salto ABK, las deportistas tuvieron un promedio de potencia de 1873,46 $\pm 231,48$ watts, el SJ $1666,13 \pm 226,17$ watts y el CMJ 1699,80 \pm 172,99 watts; y se concluyó que: No hay correlación entre la fuerza explosiva del tren inferior y la agilidad en esta población.

\section{DISCUSIÓN}

La aplicación de una revisión bibliográfica rigurosa con la finalidad de indagar e identificar la influencia del método de entrenamiento pliométrico sobre la potencia del tren inferior en deportistas del fútbol sala. A través del rastreo bibliográfico de investigaciones desarrolladas, se busca identificar las adaptaciones inducidas del método de entrenamiento pliométrico sobre la potencia del tren inferior en deportistas practicantes del fútbol sala. Esto tiene como objeto de búsqueda primordial, identificar, sí, se logra un efecto positivo en la potencia del tren inferior de los sujetos objeto de estudio. $\mathrm{Y}$, de esta manera, poder aportar a los procesos formativos de los entrenadores y dirigentes que orientan el fútbol sala.

Esta investigación a través de su producción quiere generar nuevos procedimientos técnicos y tácticos, que con bajos costos faciliten mejorar el rendimiento deportivo de los jugadores de fútbol sala, para poder incrementar las oportunidades de consecución de altos logros en estos deportistas. La finalidad de esta investigación radicaba en identificar y destacar los efectos más relevantes encontrados en las investigaciones revisadas, con la finalidad de 
brindar estrategias y procedimientos para seleccionar métodos de entrenamiento que faciliten desarrollar de manera adecuada y pertinente la potencia del tren inferior.

Los resultados se extraen de los trabajos de campo aplicados y acompañados de sus análisis estadísticos, que facilitaron obtener, la información adecuada y pertinente, en cuanto a los efectos que produce el método de entrenamiento pliométrico sobre la potencia del tren inferior de las unidades de estudio. El entrenamiento pliométrico enfocado a desarrollar la potencia del tren inferior, se debe realizar por lo menos tres veces por semana, con un día de descanso entre cada sesión como se evidencia en diversas investigaciones, y no se recomienda realizarlo un día antes y/o un día después de la competencia, para no sobrecargar muscularmente al deportista generando lesiones.

La cuantificación de la carga para dar cumplimiento de la aplicación del método de entrenamiento pliométrico como medio para fortalecer la potencia del tren inferior, debe dar cumplimiento al respeto por los principios de individualización de la carga. En las investigaciones en las que el entrenamiento pliométrico, no generó mejoras significativas, tienen como sustentación el poco tiempo de aplicación del programa, y debido también, a que los saltos se realizaban a nivel del suelo, hecho por el cual, se deduce que fueron cargas ineficaces.

\section{CONCLUSIONES}

- El entrenamiento Pliométrico resulta ser eficaz para el desarrollo de la potencia en el tren inferior, de modo que se debe aumentar el número de sesiones de entrenamiento para que los resultados tengan una mayor eficacia y resultado.

- La eficacia del sentido práctico del método de entrenamiento pliométrico, depende del proceso de entrenamiento y la adaptabilidad de los deportistas objeto de aplicación del método en mención.

- El entrenamiento pliométrico enfocado a desarrollar la potencia del tren inferior, se debe realizar por lo menos 3 veces por semana, con un día descanso entre cada sesión como se evidencia en diversas investigaciones, y no se recomienda realizarlo un día antes y/o después de la competencia, para no sobrecargar muscularmente el deportista generando lesiones.

- La cuantificación de la carga para la aplicación del método de entrenamiento pliométrico como medio para mejora la potencia del tren inferior, debe respetar los principios de individualización de la carga.

- Las investigaciones en las que el método de entrenamiento en mención, no genera mejoras significativas, se explica por el poco tiempo de aplicación del programa, y a que sus saltos se realizan a nivel del suelo, por lo que cual, puede interpretarse que son cargas ineficaces. 


\section{REFERENCIAS BIBLIOGRÁFICAS}

BOMBA, T. 2003. Entrenamiento de la potencia para el Fútbol. PubliCe Standard.

BOSCO., C., LUHTANEN, P., \& KOMI, P. 1979. A Simple Method for Measurement. Applied

Physiology, 273-282.

BROW, E., \& MAYHEW, J. 1986. Effect of plyometric training on vertical jump performance in

high school basketball players. Sports Med, 26(1), 1-4.

CERAFÍN, J. 1993. Las cualidades físicas y sus etapas sensibles: La fuerza. Sport y medicina, 15-

18.

CHU, D. 1993. Ejercicios pliométricos.

CROIN, J., \& SLEIVERT, G. 2005. Halleng in understanding the influence of maximal power training on imporving athletic performarce. Sports Medicine, 213-234.

GARCÍA, D., LÓPEZ, J. 2003. Metodología del entrenamiento pliométrico. Revista Internacional de Medicina y Ciencias de la Actividad Física y el Deporte, 14.

GARCÍA, D., ET AL. 2005. Análisis de las adaptaciones inducidas por cuatro semanas de entrenamiento pliométrico. Revista Internacional de Medicina y Ciencias de la Actividad Física y del Deporte, 68-76.

DELGADO, P. 2011. Análisis del desarrollo de la fuerza reactiva y saltabilidad en basquetbolistas que realizan un programa de entrenamiento pliométrico. Motricidad y persona, 33-44.

DIALLO, O., DORE, E., DUCHE, P., \& VAN PRAAGH, E. 2001. Effects of plyometric training followed by a reduced training programme on physical performance in prepubescent soccer players. Sports Med Phys Fitness, 41(3), 342-348.

FLARITY, J., SHILSTONE, M., IGLESIA, T., \& FISHER, Z. 1997. Effectiveness of the Strengt Shoe in enhancing sport specific skills. Recuperado el 16 de 09 de 2019, de http:// www.strengthsystems.com/strshoes.php.

GARCÍA, D., ET AL. 2003. Metodología del entrenamiento pliométrico. Revista Internacional de Medicina y Ciencias de la Actividad Física y el Deporte, 14.

GARCÍA MANSO, J. S.F. La fuerza. Editorial Gymnos.

GEMAR, J. 1988. The effects of weight training and plyometric training on vertical jump, standing long jump and forty-meter sprint. Microform Publications. College of Human Development and Performance. University of Oregon. Microform Publications., 7-22. GONZÁLEZ, J., ET AL. 2000. Concepto y medida de la fuerza explosiva en el deporte. Posibles aplicaciones al entrenamiento. Revista de entrenamiento deportivo, 5-16.

GONZÁLEZ, J. 2000. Concepto y medida de la fuerza explosiva en el deporte. Posibles aplicaciones al entrenamiento. Revista de Entrenamiento Deportivo, 5-16. 
GUTIÉRREZ, F. 2010. Concepto y clasificación de las capacidades físicas. Revista de investigación cuerpo, cultura y movimientos, 1(1), 77-86.

HÄKKINEN, K., ET AL. 1985. Effect of explosive type strength training on isometric force- and relaxation-time, electromyographic and muscle fibre characteristics of leg extensor muscles. Physiol Scand., 7(2), 587-600.

HARTMAN, J., \& TÜNNEMANN, H. S.F. Entrenamiento moderno de la fuerza. Barcelona, España: Editorial Paidotribo.

HERNÁNDEZ, Y., \& GARCÍA, J. 2012. Efectos de un entrenamiento específico de potencia aplicado a futbolistas juveniles para mejorar la velocidad lineal. Motricidad. European Journal of Human Movement, 125-144.

HERZONG, W., \& AIT- HADDOU, R. 2003. Mechanical muscle models and their application to force and power production. Strength and power in sport, 154-183.

MANCIPE, E. 2010. Métodos con pesas y pliometría: Comparación de los efectos para desarrollar potencia en jugadoras de futsal FIFA. Ef Deportes. Obtenido de http://www.efdeportes.com

MATAVULJ, D., KUKOLJ, M., UGARKOVIC, D., TIHANYI, J., \& JARIC, S. 2001. Effects of plyometric training on jumping performance in junior basketball players. Sports Med. Phys. Fitness, 41(2), 159-164.

MILLER, M., ET AL. 2016. Efectos de un Programa de Entrenamiento Pliométrico de Seis Semanas sobre la Agilidad. Sports Science \& Medicine.

MORA, V. 1995. Teoría y práctica del acondicionamiento físico. Madrid: Colegio Profesional de educación física (COPLEF).

RAMÍREZ, J. 2015. Comparación entre las respuestas de potencia muscular producidas por entrenamiento pliométrico y banda elástica en jugadores juveniles de 16 años del club Independiente Santa fe. Bogotá, D.C.: Universidad Pedagógica Nacional, p. 98.

RUÍZ DE LA CRUZ, O., \& LEAL, F. 2007. Fuerza explosiva en el futbolista profesional del Club Deportivo Independiente Medellín durante la segunda temporada competitiva del 2006, p. 106.

SIFF, M., \& VERKHOSHANSKY, Y. 2000. Super entrenamiento.

SPURRS, R., MURPHY, A., \& WATSFORD, M. 2003. The effect of plyometric training on distance running performance. Eur. J. Appl. Physiol, 89(1), 1-17.

TORRIJOS, J., ACOSTA, P., \& BENÍTEZ, D. 2018. Correlación entre la fuerza explosiva del tren inferior y la agilidad en el fútbol sala. Revista Digital: Actividad Física Y Deporte, 5(1), 15-25. https://doi.org/10.31910/rdafd.v5.n1.2019.1120

VALERO, D., \& SUÁREZ, M. 2018. Análisis de la evaluación de potencia en tren inferior: Una revisión sistemática. Actividad física y deporte, p. 33.

VILLAR, A. 1985. La preparación física del fútbol basada en el atletismo. En Villar, C. La preparación física del fútbol basada en el atletismo. Madrid: S.L. Gymnos, p. 841. 
WILSON, G., NEWTON, R., MURPHY, A., \& HUMPHRIES, B. 1993. The optimal training load for the developement of dynamic athletic performance. Med. Sci. Sports Exerc, 25(11), 12791286.

ZATSIORSKI, V. 1989. Metodología deportiva, p. 229.

Cómo citar: Cepeda, C., Gamboa, F., \& Sanabria, Y. (2019). Antecedentes, descripción, potencia del tren inferior y pliometría en fútbol sala. R. Actividad fis. y deporte. 6 (1): 165-178.

Artículo de acceso abierto publicado por: Revista Digital: Actividad Física y Deporte, bajo una licencia Creative Commons CC BY-NC 4.0. 